\title{
Módulo de elasticidade aparente em vigas roliças estruturais de madeira Pinus elliottii
}

\author{
Apparent modulus of elasticity in Pinus elliottii structural \\ round timber beams
}

\author{
André Luiz Zangiácomo \\ André Luis Christoforo \\ Francisco Antonio Rocco Lahr
}

\section{Resumo}

André Luiz Zangiácomo Universidade Federal de Lavras Lavras - MG - Brasil

Andrá Luis Christoforo Universidade Federal de Sâo Carlos São Carlos - SP - Brasil

Francisco Antonio Rocco Lahr Universidade de São Paulo São Carlos - SP - Brasil

Recebido em 08/12/12 Aceito em 22/02/13

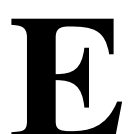

ste trabalho investiga, com o auxílio da norma brasileira ABNT NBR 7190:1997 (Projeto de Estruturas de Madeira) e do ensaio de flexão estática a três pontos, a influência das medidas entre o comprimento (L) e o diâmetro (d) de peças estruturais de madeira roliça da espécie Pinus elliottii no cálculo do módulo de elasticidade (MOE). Foram ensaiadas, de forma não destrutiva, 24 peças de madeira (verde) com comprimento médio de $750 \mathrm{~cm}$ e diâmetro médio de $30 \mathrm{~cm}$. Para tanto, foram determinados os valores dos módulos de elasticidade para seis relações (L/d) distintas entre comprimento e diâmetro (9; $12 ; 15 ; 18 ; 21 ; 24)$, fornecendo seis valores distintos de MOE por peça utilizada. Os resultados da análise de variância indicaram equivalências dos módulos de elasticidade apenas entre as relações L/d iguais a 24, 21, 18 e 15, revelando ser de 15 o menor valor da relação responsável por minorar significativamente o efeito das forças cisalhantes. Isso implica que, para o uso adequado da equação de cálculo do módulo de elasticidade pela norma brasileira para as peças de roliças de madeira Pinus elliottii, faz-se necessário respeitar a relação $\mathrm{L} / \mathrm{d} \geq 15$.

Palavras-chave: Rigidez. Flexão.Teoria de vigas.

\section{Abstract}

This study investigates the influence of the measurements between the length $(L)$ and diameter (d) of Pinus elliottii round timber structures to determine the modulus of elasticity (MOE), using the Brazilian standard ABNT NBR 7190:1997 (Design of Wood Structures) and the three-point static bending test. 24 pieces of green wood with an average length of $750 \mathrm{~cm}$ and average diameter of $30 \mathrm{~cm}$ were tested nondestructively. In order to do that, the values of the modulus of elasticity for six different ratios $(L / d)$ between length and diameter $(9,12,15,18,21,24)$ were determined, producing six different values of MOE for each structural element. The ANOVA results indicated equivalence of the modulus of elasticity only between the L/d relations equal to $24,21,18$ and 15, where 15 proved to be the smallest value able to alleviate the shear effect. This implies that for the proper use of the equation to obtain the elastic modulus by the Brazilian standard for Pinus elliiottii structural round timber, it is necessary to respect the $L / d \geq 15$ relation.

Keywords: Stiffness. Bend. Beams theory. 


\section{Introdução}

A disponibilidade da madeira associada a novas tecnologias contribui para o aumento de sua utilização em estruturas (LARSON; MIRTH; WOLFE, 2004; MINÁ, 2005; BRITO; CALIL, 2010; CHRISTOFORO et al., 2011a), com ênfase na construção civil. Neste setor, a madeira pode ser empregada na forma serrada ou em sua forma roliça natural.

As peças roliças representam um dos usos mais eficientes dos recursos florestais, pois requerem um mínimo de processamento entre o corte da árvore e a comercialização da peça (CHRISTOFORO et al., 2012). Os elementos são descascados, secados e, muitas vezes, é feito um tratamento com preservativos antes da aplicação estrutural. Existem diversas possibilidades do uso desse material, destacando-se entre elas as estruturas para pontes, as estruturas aplicadas às redes elétricas aéreas de distribuição (postes e torres), pilares, estacas de fundações, habitações, coberturas e estruturas de contenção (ZANGIÁCOMO, 2007).

A norma brasileira NBR 7190 (ABNT, 1997) especifica os elementos roliços a partir dos diâmetros de base e de topo, independentemente da espécie utilizada. $\mathrm{O}$ mesmo documento determina que as propriedades de resistência e rigidez sejam obtidas em ensaios em corpos de prova de pequenas dimensões e isentos de defeitos, mesmo sendo conveniente o uso da peça com dimensões estruturais (MINÁ; DIAS, 2008).

Os corpos de prova para a obtenção das propriedades de resistência e rigidez da madeira na flexão pela norma NBR 7190 (ABNT, 1997) possuem a dimensão $5 \mathrm{~cm} \times 5 \mathrm{~cm} \times 110 \mathrm{~cm}$, respeitando-se a relação entre comprimento $(L)$ e largura da seção $(h)$ igual a 21 vezes. A relação $L / h \geq 21$ foi obtida, entre outros resultados, do trabalho de Rocco Lahr (1983), utilizando-se do ensaio de flexão estática a três pontos em peças de madeira serrada. Os ensaios executados na mesma peça (não destrutivos) diferenciaram-se apenas pelas aproximações sucessivas dos apoios, possibilitando investigar a influência de relações $L / h$ distintas no cálculo do módulo de elasticidade, obtidos com o auxílio da Equação 1, que contabiliza apenas a existência de esforços fletores, sendo $\delta$ o deslocamento no ponto médio, $F$ o carregamento aplicado no meio do vão, $L$ o vão entre apoios, $E_{M}$ o módulo de elasticidade e $I$ o momento de inércia da seção transversal. Os valores obtidos dos módulos de elasticidade com o uso da Equação 1 apresentaram ser equivalentes (convergentes) para relações $L / h \geq 21$, e distintos para relações inferiores, intitulados aparentes $\left(\mathrm{E}_{\mathrm{ap}}\right)$.
Esse resultado garante que os esforços cisalhantes presentes no ensaio de flexão podem ser desprezados na determinação dos deslocamentos, validando o modelo de cálculo (teoria de vigas de Euller Bernoulli) utilizado pela norma brasileira NBR 7190 (ABNT, 1997) e por outros códigos normativos para a determinação do módulo de elasticidade.

$$
E_{m}=\frac{F \cdot L^{3}}{48 \cdot \delta \cdot I}
$$

No Brasil, as normas vigentes que tratam de elementos roliços (NBR 6231 (ABNT, 1980); NBR 8456 (ABNT, 1984a); NBR 8457 (ABNT, 1984b); NBR 6122 (ABNT, 1996)) estão há pelo menos 20 anos sem revisão técnica (CHRISTOFORO, 2011b), e trabalhos envolvendo a caracterização de elementos roliços em geral fazem uso das metodologias de cálculo contidas em documentos normativos, não sendo o foco dessas pesquisas o estudo das dimensões dos elementos roliços para o adequado emprego da teoria de vigas responsável por fornecer o módulo de elasticidade das peças (ZANGIÁCOMO, 2007).

Este trabalho investiga, com o auxílio do ensaio de flexão estática a três pontos e da norma brasileira NBR 7190 (ABNT, 1997), adaptada para peças de dimensões estruturais, a influência da relação entre o comprimento das peças e o diâmetro médio (medido no ponto de aplicação da força) responsável por anular o efeito das forças cisalhantes para o correto uso da equação para o cálculo do módulo de elasticidade pela norma brasileira.

\section{Material e métodos}

As peças de madeira roliça da espécie Pinus elliottii (madeira verde-saturada) foram doadas (24 exemplares) pela Estação Ecológica de Itirapina da Secretaria do Meio Ambiente do Estado de São Paulo, com comprimento médio de $750 \mathrm{~cm}$, diâmetro médio de $30 \mathrm{~cm}$, densidade de $0,55 \mathrm{~g} / \mathrm{cm}^{3}$ e conicidade média de $4 \%$.

A metodologia empregada na obtenção da relação $L / d$ (comprimento por diâmetro) nas 24 peças de madeira roliça foi a mesma utilizada no trabalho de Rocco Lahr (1983), sendo os ensaios de flexão a três pontos executados nas dependências do Laboratório de Madeiras e de Estruturas de Madeira (LaMEM) do Departamento de Engenharia de Estruturas (SET) da Escola de Engenharia de São Carlos (EESC/USP).

As relações entre comprimento e diâmetro investigadas foram $24,21,18,15,12$ e 9,

8 Zangiácomo, A. L.; Christoforo, A. L.; Rocco Lahr, F. A. 
conduzindo a um total de 6 experimentações por peça roliça e 144 ensaios ao todo. Para garantia de linearidade física e geométrica (ensaios não destrutivos) das peças testadas, os deslocamentos no meio do vão foram restritos à medida $L / 200$, como preconiza a norma brasileira NBR 7190 (ABNT, 1997). Cabe ressaltar que os ensaios experimentais foram realizados sequencialmente, iniciados pelas peças de maior relação $L / d(24)$, e consequentemente de maior comprimento, seguido pelas demais relações $(21,18,15,12$ e 9$)$.

O módulo de elasticidade das peças roliças foi obtido de acordo com a Equação 1, e o cálculo do momento de inércia da peça foi feito considerando o valor obtido da seção circular medida no ponto médio das vigas (simplificação). Essa simplificação está de acordo com os resultados obtidos do trabalho de Christoforo et al. (2011a), que investigaram, com o auxílio do método dos elementos finitos e do método dos mínimos quadrados, a influência da geometria troncocônica de peças roliças estruturais de eucalipto no cálculo do módulo de elasticidade na flexão, comparando os resultados com uma metodologia simplificada, adaptada da norma brasileira NBR 7190 (ABNT, 1997), utilizando-se o diâmetro do ponto médio da peça no cálculo do momento de inércia. Os módulos de elasticidade de ambas as formas de cálculo, simplificada e numérica, apresentaram equivalência estatística, implicando, para as madeiras avaliadas, a boa aproximação do modelo simplificado de cálculo proposto pela norma brasileira.

Para verificação da equivalência ou não entre as médias dos valores dos módulos de elasticidade obtidos das relações $L / d$ investigadas foram utilizados testes de hipótese (Teste t), com nível de significância de 5\% (95\% de confiabilidade), executados com o auxílio do software Minitab ${ }^{\mathrm{TM}}$ versão 14. Quando, para os graus de liberdade e significância definidos, o parâmetro $F_{\text {calc calculado }}$ pelo software é menor que o parâmetro tabelado $F_{\text {tab}}$, aceita-se a hipótese nula $H_{0}$ (as médias populacionais são iguais), ou, ainda, se o P-valor for menor que o nível de significância $\alpha$, rejeita-se a hipótese nula (MONTGOMERY, 2005). Como forma de validar os resultados obtidos do uso do teste de hipótese, foram verificadas a normalidade e a homogeneidade da variância dos dados, com o auxílio do teste de Ryan-Joyner e Bartlett, respectivamente. $\mathrm{O}$ nível de significância estipulado para os referidos testes foi de 5\%, consistindo em normalidade dos dados e homogeneidade entre variâncias como hipótese nula, e a não normalidade e não equivalência entre variâncias como hipótese alternativa. P-valor do teste superior ao nível de significância implica aceitar $H_{0}$, refutando-a em caso contrário.

\section{Resultados}

Na Tabela 1 são apresentados os valores médios $\left(\mathrm{X}_{\mathrm{m}}\right)$, desvios padrão (DP), coeficientes de variação (CV) e os valores máximo (Máx) e mínimo (Mín) dos módulos de elasticidade aparentes $\left(\mathrm{E}_{\mathrm{ap}}\right)$ para cada relação $L / d$ investigada.

A Figura 1 ilustra o diagrama força×deslocamento para uma das peças ensaiadas, em que cada reta corresponde a uma das relações $L / d$ investigadas.

A Figura 2 ilustra o gráfico das médias dos módulos de elasticidade aparentes obtidos das relações $L / d$. Uma linha de tendência entre os valores médios (discretos) foi ajustada apenas para evidenciar o decréscimo nas diferenças entre dois valores médios sucessivos com o aumento da relação $L / d$.

Na Tabela 2 são apresentados os resultados correspondentes à análise de variância (ANOVA) dos dados das médias dos módulos de elasticidade aparentes obtidos entre as diferentes relações $L / d$. Os gráficos do teste de normalidade (Ryan-Joyner) de resíduos versus valores estimados e da homogeneidade entre variâncias para os valores de $\mathrm{E}_{\text {ap }}$ em função das relações $L / d$ são ilustrados nas Figuras 3, 4 e 5, respectivamente

Tabela 1 - Módulos de elasticidade aparentes (MPa) para as 6 relações $L / d$

\begin{tabular}{c|c|c|c|c|c|c}
\hline & \multicolumn{6}{|c}{ Módulos de elasticidade aparentes $\left(\mathbf{E}_{\mathbf{a p}}\right)$ - MPa } \\
\hline Estatísticas & L/d = 24 & L/d = 21 & L/d = 18 & L/d = 15 & L/d = 12 & L/d = 9 \\
\hline $\boldsymbol{X}_{\boldsymbol{m}}$ & 10759 & 10731 & 10284 & 9594 & 8765 & 6762 \\
$\boldsymbol{D P}$ & 2201 & 2234 & 1999 & 1749 & 1326 & 941 \\
$\boldsymbol{C V}(\boldsymbol{\%})$ & 20 & 21 & 19 & 18 & 15 & 14 \\
Mín & 7127 & 6711 & 6961 & 6635 & 6416 & 5075 \\
Máx & 15042 & 14236 & 13731 & 12615 & 10853 & 8361 \\
\hline
\end{tabular}


Figura 1 - Diagrama forçaxdeslocamento de uma peça para as 6 relações $L / d$

\section{Diagrama força $\times$ deslocamento}

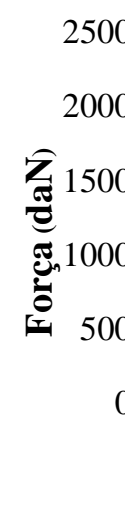

(3)

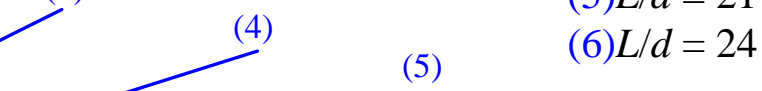

Figura 2 - Valores médios dos módulos de elasticidade aparentes versus relações $L / d$

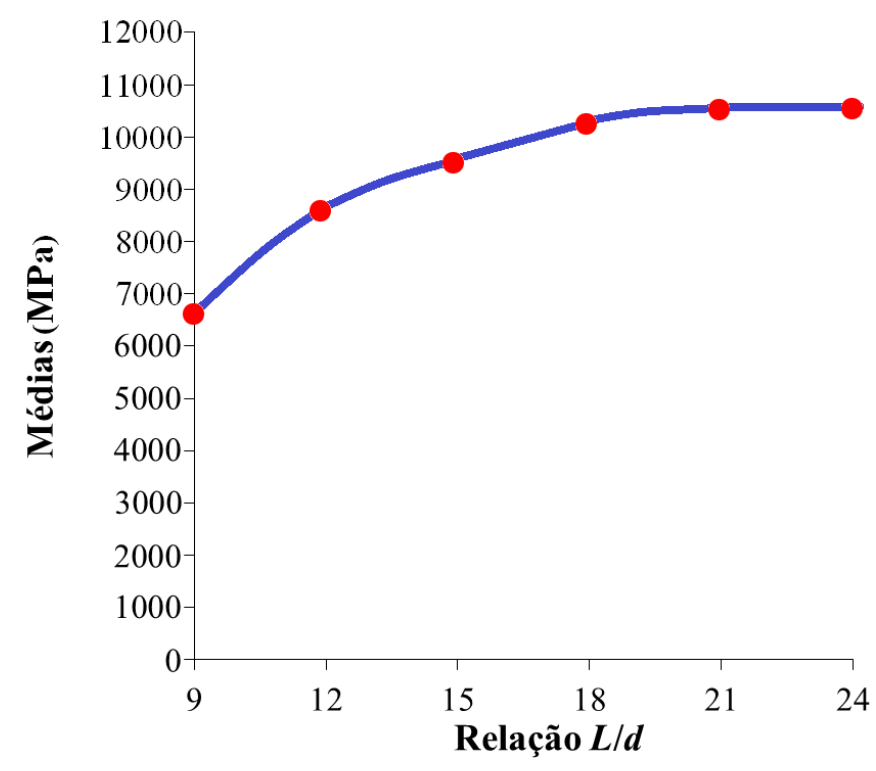

Tabela 2 - Resultados da análise de variância dos módulos de elasticidade entre as relações $L / d$

\begin{tabular}{l|c|c|c}
\hline \multicolumn{1}{c|}{ Relações $(\mathbf{L} / \boldsymbol{d})$} & $\mathbf{F}_{\text {calc }}$ & $\mathbf{F}_{\text {tab }}$ & P-valor \\
\hline $24 \times 21$ & 0,00 & 4,05 & 0,965 \\
$24 \times 21 \times 18$ & 0,37 & 3,14 & 0,692 \\
$24 \times 21 \times 18 \times 15$ & 1,68 & 2,72 & 0,176 \\
$24 \times 21 \times 18 \times 15 \times 12$ & 4,63 & 2,46 & 0,002 \\
$24 \times 21 \times 18 \times 15 \times 12 \times 9$ & 17,34 & 2,29 & 0,000 \\
\hline
\end{tabular}

10 Zangiácomo, A. L.; Christoforo, A. L.; Rocco Lahr, F. A. 
Figura 3 - Gráfico do teste de normalidade de Ryan-Joyner para as relações $L / d$

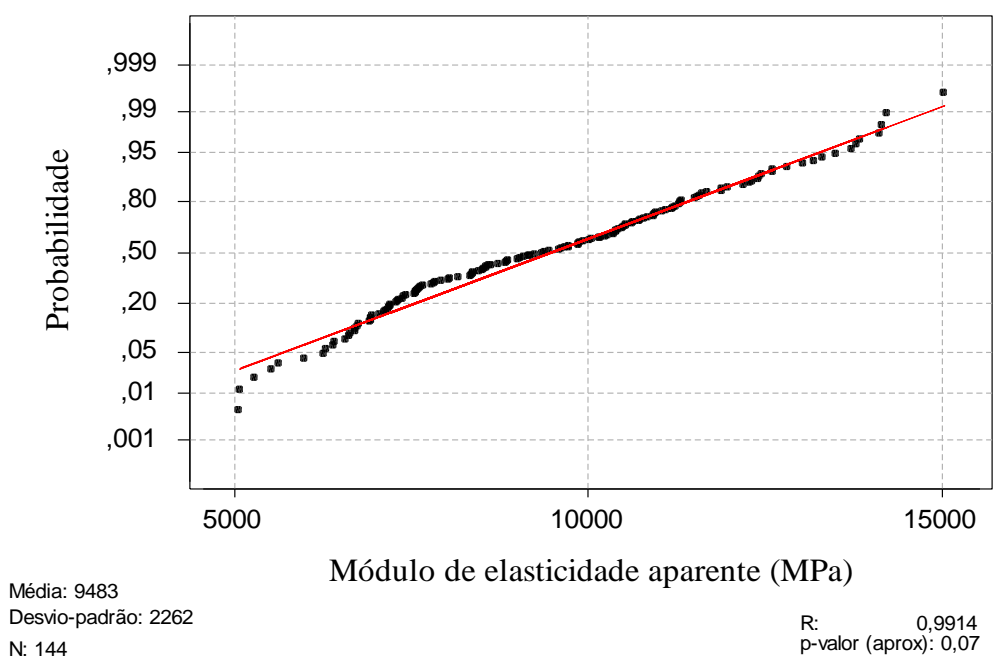

Figura 4 - Gráfico dos resíduos versus valores estimados para as relações $L / d$

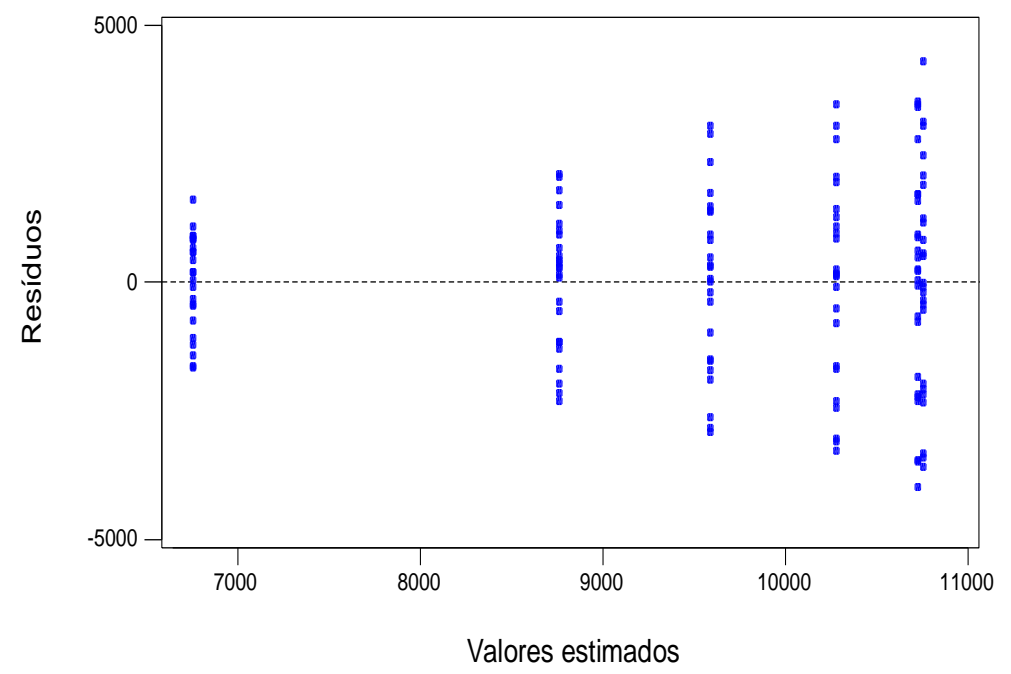

Figura 5 - Resultado do teste de homogeneidade entre variâncias

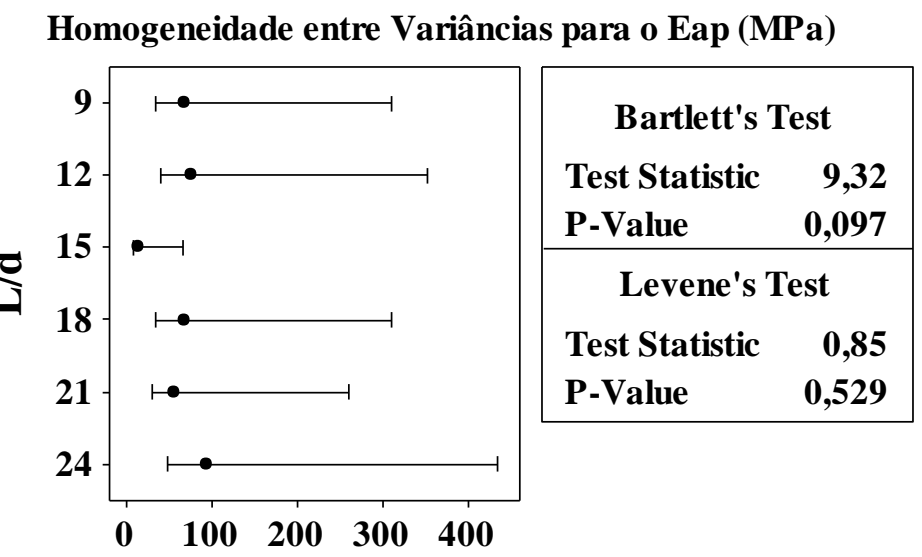

Intervalo de Confiança de Bonferroni (95\%) 
Os resultados apresentados nas Figuras 3, 4 e 5 validam o modelo da ANOVA. Os dados apresentados na Tabela 2 revelaram que as médias referentes às relações $L / d=24,21,18$ e 15 são estatisticamente equivalentes, a um nível de significância de 5\%, apresentando $\mathrm{F}_{\text {calc }}$ menores que os $\mathrm{F}_{\mathrm{tab}}$ e $\mathrm{P}$-valores maiores que 0,05. Apontam ainda que, ao serem inseridas as relações $L / d=12$ e 9, as médias tornam-se estatisticamente diferentes, com as análises das respectivas apresentando valores de $\mathrm{F}_{\text {calc }}$ maiores que os $\mathrm{F}_{\text {tab }}$ (4,63 sendo maior que 2,46 e 17,34 também maior que 2,29), além de P-valores menores que 0,05. Dessa forma, a menor relação $L / d$ investigada responsável por minorar de forma significativa a influência do cisalhamento nos ensaios de flexão foi a de 15 .

\section{Conclusões}

O resultado encontrado para as madeiras roliças da espécie Pinus elliottii apontou ser válido o uso da Equação 1 apenas para relações $L / d \geq 15$, sendo diferente da relação apresentada pela norma brasileira NBR 7190 (ABNT, 1997) para peças de madeira serrada $(L / h \geq 21)$. Isso implica que, respeitado o limite da relação $L / d$ aqui encontrada, o ensaio de flexão estática a três pontos possibilita obter de modo confiável o módulo de elasticidade à flexão das peças, sabendo ser desprezível a influência do esforço cortante nessas condições.

\section{Referências}

ASSOCIAÇÃO BRASILEIRA DE NORMAS TÉCNICAS, NBR 6122: projeto e execução de fundações. Rio de Janeiro, 1996.

ASSOCIAÇÃO BRASILEIRA DE NORMAS TÉCNICAS. NBR 6231: postes de madeira: resistência à flexão. Rio de Janeiro, 1980.

ASSOCIAÇÃO BRASILEIRA DE NORMAS TÉCNICAS. NBR 7190: projeto de estruturas de madeira. Rio de Janeiro, 1997.

ASSOCIAÇÃO BRASILEIRA DE NORMAS TÉCNICAS. NBR 8456: postes de eucalipto preservado para redes de distribuição de energia elétrica. Rio de Janeiro, 1984a.

ASSOCIAÇÃO BRASILEIRA DE NORMAS TÉCNICAS. NBR 8457: postes de eucalipto preservado para redes de distribuição de energia elétrica: dimensões. Rio de Janeiro. 1984b.
BRITO, L. D.; CALIL JR., C. Manual de Projeto e Construção de Estruturas Com Peças Roliças de Madeira de Reflorestamento. Cadernos de Engenharia de Estruturas, São Carlos, v. 12, n. 56, p. 57-77, 2010.

CHRISTOFORO, A. L. et al. Numerical Evaluation of the Modulus of Longitudinal Elasticity in Structural Round Timber Elements of the Eucalyptus genus. Engenharia Agrícola, Jaboticabal, v. 31, p. 1007-1014, 2011 a.

CHRISTOFORO, A. L. et al. The Position Effect of Structural EucalyptusRound Timber on the Flexural Modulus of Elasticity.Engenharia Agrícola, Jaboticabal, v. 31, p. 1219-1225, 2011 b.

CHRISTOFORO, A. L. et al.Numerical

Evaluation of Longitudinal Modulus of Elasticity of Eucalyptus grandis Timber

Beams.International Journal of Agriculture and Forestry, v. 2, p. 166-170, 2012.

LARSON, D.; MIRTH, R.; WOLFE, R. Evaluation of Small-Diameter Ponderosa Pine Logs in Bending.Forest Products Journal, Madison, v. 54, n. 12, p. 52-58, dez. 2004.

MINÁ, A. J. S. Estudo de Estacas de Madeira Para Fundações de Pontes de Madeira. $117 \mathrm{f}$. São Carlos, 2005. Tese (Doutorado em Engenharia de Estruturas) - Escola de Engenharia,

Universidade de São Paulo, São Carlos, 2005.

MINÁ, A. J. S.; DIAS, A. A. Estacas de Madeira Para Fundações de Pontes de Madeira. Cadernos de Engenharia de Estruturas, v. 10, p. 129-155155, 2008.

MONTGOMERY, D. C. Design and Analysis of Experiments. $6^{\text {th }}$. ed. Arizona: John Wiley \& Sons, 2005.

ROCCO LAHR, F. A. Sobre a Determinação de Propriedades de Elasticidade da Madeira. $221 \mathrm{f}$. São Carlos. 1983. Tese (Doutorado em Engenharia de Estruturas) - Escola de Engenharia, Universidade de São Paulo, São Carlos, 1983.

ZANGIÁCOMO, A. L. Estudo de Elementos Estruturais Roliços de Madeira. 136 f. São Carlos, 2007. Tese (Doutorado em Engenharia de Estruturas) - Escola de Engenharia, Universidade de São Paulo, São Carlos, 2007. 


\section{André Luiz Zangiácomo}

Departamento de Engenharia | Universidade Federal de Lavras | Campus Universitário | Lavras - MG - Brasil | Caixa Postal 3037 | CEP 37200-000 | Tel.: (35) 3829-5117 Ramal 5117 | E-mail: andrezangiacomo@deg.ufla.br

\section{Andrá Luis Christoforo}

Departamento de Engenharia Civil | Universidade Federal de Sâo Carlos | Campus São Carlos | São Carlos - SP - Brasil | CEP: 13565-905 | Tel.: (16) 3351-8262 | Email: alchristoforo@yahoo.com.br

\section{Francisco Antonio Rocco Lahr}

Escola de Engenharia de São Carlos | Universidade de São Paulo | Av. Trabalhador Sãocarlense, n. 400, Centro | São Carlos - SP - Brasil | CEP 13566-590 | Tel.: (16) 3373-8206 | Email: frocco@sc.usp.br

\section{Revista Ambiente Construído}

Associação Nacional de Tecnologia do Ambiente Construído

Av. Osvaldo Aranha, $99-3^{\circ}$ andar, Centro

Porto Alegre - RS - Brasil

CEP $90035-190$

Telefone: +55 (51) 3308-4084

Fax: +55 (51) 3308-4054

www.seer.ufrgs.br/ambienteconstruido

E-mail: ambienteconstruido@ufrgs.br 